\title{
Influence of morphological parameters of a 2D random short fibre composite on its effective elastic properties
}

\author{
W. Leclerc ${ }^{1,2}$, P. Karamian-Surville ${ }^{1,2, a}$ And A. Vivet ${ }^{3,4,5,6}$ \\ 1 Université de Caen Basse-Normandie, UMR 6139 LMNO, 14032 Caen, France \\ 2 CNRS, UMR 6139 LMNO, 14032 Caen, France \\ 3 Université de Caen Basse-Normandie, UMR 6252, 14032 Caen, France \\ 4 ENSICAEN, UMR 6252, CIMAP, 14050 Caen Cedex 4, France \\ 5 CNRS, UMR 6252, 14032 Caen, France \\ ${ }^{6}$ CEA, UMR 6252, 14032 Caen, France
}

Received 14 September 2013, Accepted 18 November 2013

\begin{abstract}
The paper deals with the assessment of the influence of morphological parameters of a $2 \mathrm{D}$ random short fibre composite on its effective elastic properties. A double-scale 2D finite element approach is used and periodic representative volume elements are generated according to the model with an $n$-order approximate geometry. This one is a recent and powerful tool to generate meshes in the context of complex microstructures such as random fibre composites. Impact of three morphological features namely, aspect ratio, tortuosity of fibres and width of the interphase area, is investigated. Numerical results are provided for several contrasts of properties and volume fractions of fibres.
\end{abstract}

Key words: Computational solid mechanics / short fibre composites / RVE / finite element analysis (FEA)

\section{Introduction}

For several decades short fibre composites have been manufactured and used for industrial purposes. Their low cost of production associated to their excellent strengthto-weight ratio have led to numerous applications in many fields like aerospace, automotive engineering, and competitive sport. For instance, fibreglass is massively produced for building hulls in marine industry and carbonfibre-reinforced composites are used for the retrofitting of old structures in civil engineering. The main issue is to assess their intrinsic mechanical properties in order to evaluate their ability to replace one-phase materials. Experimental measurements are sometimes difficult and costly to set up for this purpose. That is why analytical models are very useful for estimating effective properties. Mori-Tanaka micromechanics model [1] and self-consistent estimates [2] are classical tools for predicting elastic coefficients of two-phase heterogeneous media. However, accurate predictions of the mechanical response are difficult to obtain since this one strongly depends on several parameters such as orientation, shape and aspect ratio of heterogeneities. Thus, numerous micromechanics models have been proposed during the last decades for

\footnotetext{
a Corresponding author: philippe.karamian@unicaen.fr
}

improving the accuracy of estimates, among which, the general self-consistent scheme [3] and the third-order approximation [4].

However, the range of validity of a micromechanics model is always restricted. Thus, there is no model for complex-shaped heterogeneities, for example tortuous fibres, for which no Eshelby's tensor is available. In this context, a numerical modelling is more convenient to evaluate effective properties. The basic idea consists in generating a Representative Volume Element (RVE) of the material either by a Random Sequential Adsorption (RSA) [5] for which distance constraints prevent the overlap between two or more heterogeneities, or a fully MonteCarlo (MC) process [6]. Effective elastic properties are generally evaluated either with the help of a Fast Fourier Transformation [7], or more classically, by Finite Element (FE) analysis [8]. Unfortunately, in the context of random heterogeneous media, a FE approach is difficult to set up since the mesh generation is thwarted by several drawbacks related to the complex structure of the network of heterogeneities. Hence, a modelling of such a microstructure is often basic and unrealistic. However, the recent development of the model with an $n$-order approximate geometry [9] which is based on a grid approximation, has opened prospects for the generation of complex 


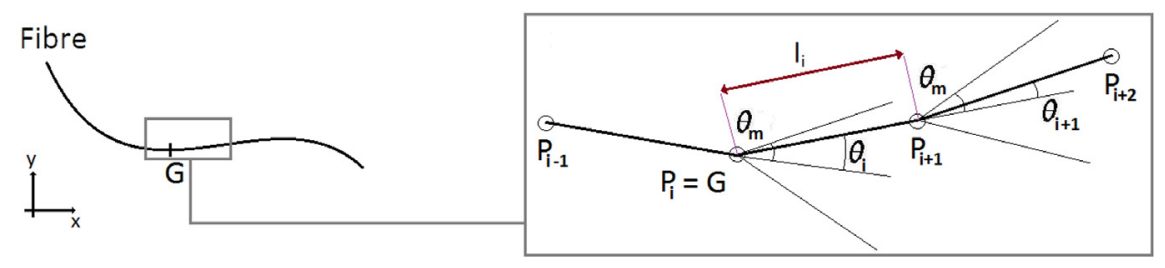

Fig. 1. Parametrisation of the tortuosity of a fibre.

microstructures. In the present work, a FE approach using this concept is considered in order to assess elastic coefficients of a $2 \mathrm{D}$ random short fibre composite for which the microstructure is complex and strongly entangled. Influence of three morphological parameters namely, aspect ratio, tortuosity of fibres and width of the interphase area, is investigated.

\section{Numerical modelling}

\subsection{Morphological parameters}

2D microstructures are generated according to a set of random morphological parameters describing the complex geometry of a random fibre composite. Each parameter is either randomly drawn according to a given distribution law such as, for instance, the Gaussian law and uniform law, or a fixed one. On the one hand, both centroid spatial location and orientation of each heterogeneity are randomly drawn. One must keep in mind that we assume no distance constraint between two or more fibres so that the process can be seen as a MC one. Thus, all distribution laws are unbiased, and the overlap between two or more fibres is possible. On the other hand, other parameters are set at given values in order to be investigated in a deterministic way,

- the aspect ratio $a_{r}$ of fibres which is the ratio between the length $l$ and the diameter $d$ of each heterogeneity;

- the parameter of tortuosity of fibres which describes the level of non-straightness of a heterogeneity. This one is defined by a maximum angle $\theta_{m}$ which has to be respected in the generation of a fibre in the same way as [10]. Figure 1 illustrates how one set up a tortuous fibre. The process consists in building the reinforcement point by point starting from its centroid $G$. Thus, each new point $P_{i+1}$ is obtained from its predecessors $P_{i}$ and $P_{i-1}$ considering the distance between two consecutive nodes $l_{i}$, and an angle $\theta_{i}$ which is uniformly drawn between $-\theta_{m}$ and $\theta_{m} . l_{i}$ is chosen constant and sufficiently small to ensure the smoothness of the heterogeneity;

- the width $w_{\text {int }}$ of the interphase area. This one is an area surrounding each reinforcement where the properties of the matrix are disrupted due to the presence of the heterogeneity. One must take care of not confusing interphase with interface which designates the boundary between soft and hard phases. In the present work, no decohesion is taken into account and perfect bondings are supposed.

\subsection{RVE}

The investigation of the dimensions of the RVE is a key issue from which representativeness, efficiency and validity of the numerical modelling strongly depend [11]. The size of an RVE is defined as $s$, the scale ratio between its width and the average length of each heterogeneity. $s$ must be greater than a critical scale ratio $s_{r}$ to check out the representativeness of a unit cell and avoid a bias introduced by the boundary conditions. Its assessment is realised according to the process described by [11] which leads to a compromise between accuracy of results and low cost of calculation. In the present work, an RVE is generated from the set of random and deterministic parameters described in Section 2.1. In addition, a specific treatment is performed to ensure the periodicity of the cell. Its principle consists in duplicating and translating the fibres intersecting the boundaries of the unit cell.

\subsection{Mesh generation}

A reliable, efficient and automated mesh generation of overlapping random heterogeneous media is particularly difficult to set up in a classical approach for which the mesh is directly generated from the boundaries of the network of fibres. The voxel-based FE approach [12] circumvents the drawbacks relative to the mesh generation. This one consists in approximating the boundaries of the network of fibres according to a grid of pixels in $2 \mathrm{D}$ or voxels in $3 \mathrm{D}$. The concept is very suitable for a reliable and automated mesh generation but is poorly efficient. The last point is drastically improved in the context of a strongly entangled microstructure when using the model with an $n$-order approximate geometry [9]. This one is based on a 2-step mesh generation. First, one has to generate a grid of coarse elements the size of which is typically equal or greater than the diameter of fibres. Second, an adaptive mesh refinement (AMR) is performed in order to locally refine the boundaries of each heterogeneity according to an $n$-order parameter. This one is defined as the ratio between the diameter of fibres and the size of the smallest voxel, expressed in logarithm in base 2. Thus, a Delaunay triangulation constrained to the approximate boundaries enables a reliable and automated mesh generation. In addition, it was exhibited [9] that the $n$-order strategy leads to a tremendous reduction of the calculation cost in comparison with a classical approach without AMR. Figure 2 illustrates an RVE obtained according 


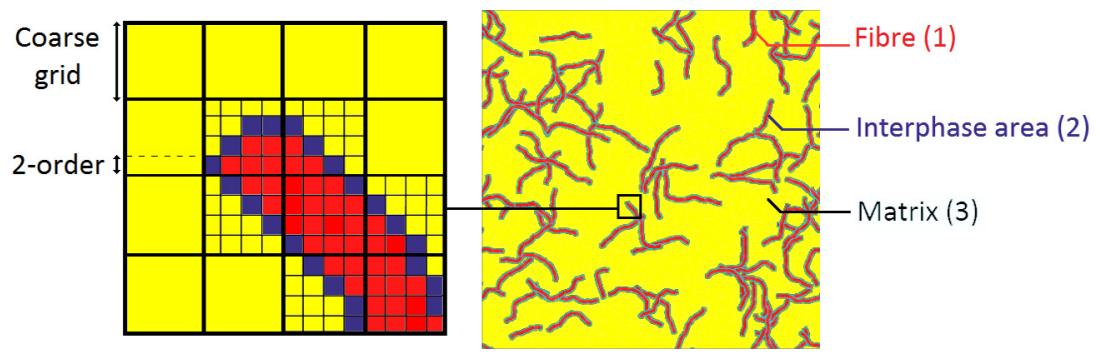

Fig. 2. RVE obtained according to the model with a 2-order approximate geometry (100-by-100 coarse grid).

to the model with a 2-order approximate geometry before meshing. The red phase (1) corresponds to the reinforcement, the blue one (2) corresponds to the interphase area and the yellow one (3) corresponds to the matrix phase. Morphological parameters are as follows, $w_{\text {int }}$ is set at $d / 4, a_{r}$ is set at $20, \theta_{m}$ is set at $40^{\circ}$ and $s$ is set at 5 . The number of heterogeneities is set at 100 .

\section{Influence of morphological parameters}

An investigation of a set of morphological parameters, namely the aspect ratio, the tortuosity and the width of the interphase is set up under the following hypotheses. First, effective elastic properties are evaluated according to the asymptotic process described by [11]. This one consists of generating a complete sample of RVEs for which elastic coefficients are assessed by a homogenisation technique namely, in the present work, the double-scale approach [13]. Effective properties are then obtained from averaging the scope of results. Second, we consider networks of randomly distributed and oriented fibres. Each one follows an isotropic transverse behaviour law and the soft phase is isotropic. $Y_{l}^{f}, Y_{t}^{f}, \mu^{f}$ and $\kappa^{f}$ designate longitudinal and transverse Young's, shear and bulk moduli of each fibre respectively. $Y^{m}, \mu^{m}$ and $\kappa^{m}$ are Young's, shear and bulk moduli of the matrix respectively. Poisson's ratios are equal to 0.25 and 0.35 for hard and soft phases respectively. In addition, we set $Y_{l}^{f}=2 Y_{t}^{f}$. $\kappa^{*}$ is the normalised effective planar bulk modulus which is estimated as follows,

$$
\kappa^{*}=\frac{1}{4 \kappa^{m}} \sum_{\alpha, \beta \leq 2} C_{\alpha \alpha \beta \beta}
$$

where $C_{\alpha \beta \gamma \delta}\left(\alpha, \beta, \gamma, \delta \in\{1,2\}^{4}\right)$ is the effective stiffness tensor. In addition, interphase area is supposed isotropic with shear and Young's moduli equal to 0.5 times the ones of the soft phase. The contrast of properties $c_{r}$ between hard and soft phases is defined as follows,

$$
c_{r}=\frac{Y_{l}^{f}}{Y^{m}}=\frac{\mu^{f}}{\mu^{m}}
$$

Finally, meshes are built according to the model with a 2-order approximate geometry, and the size of RVE $s$ is set at 5 according to previous investigations [9].

\subsection{Aspect ratio}

First, a study is realised for a range of aspect ratios $a_{r}$ between 5 and $50 . \theta_{m}$ is equal to 20 and $w_{\text {int }}$ is equal to $d / 4$. Several volume fractions of fibres are considered between 5 and 25\%. Figure 3a illustrates the influence of the aspect ratio on the normalised effective bulk modulus $\kappa^{*}$ for a contrast of properties set at 10 . One can notice that the greater the aspect ratio is the greater the bulk modulus is whatever the load rate is. Thus, $\kappa^{*}$ varies from 1.2690 to 1.4529 , for a volume fraction of fibres of $25 \%$, when the aspect ratio varies from 5 to 50 . Results are similar with higher values in the case of a high contrast of properties (Fig. $3 \mathrm{~b}, c_{r}=50$ ) for which $\kappa^{*}$ varies from 1.5171 to 2.4033 for a volume fraction of fibres of $25 \%$. One can notice that the saturation is almost reached when $c_{r}$ is equal to 10 while this one is barely noticeable for a high-contrast composite. This result is related to end effects which much more impact the mechanical response of the material when the contrast is important.

\subsection{Tortuosity}

Second, the influence of the tortuosity of fibres is examined. Maximum angle $\theta_{m}$ is set at several values between 0 for which fibres are straight and $40^{\circ}$ for which fibres are strongly tortuous. $a_{r}$ is set at $20, w_{\text {int }}$ is set at $d / 4$ and several volume fractions of fibres are considered between 5 and $25 \%$. Figure 4 a illustrates the influence of the tortuosity of fibres on $\kappa^{*}$ for $c_{r}$ set at 10 . On the one hand, results highlight a slight negative impact of the tortuosity for a low contrast of properties. Thus, $\kappa^{*}$ varies from 1.4226 to 1.3767 when $\theta_{m}$ varies from $0^{\circ}$ to $40^{\circ}$ and the volume fraction of fibres is $25 \%$. In addition, $\kappa^{*}$ varies from 1.10587 to 1.0676 when $\theta_{m}$ varies from $0^{\circ}$ to $40^{\circ}$ and the volume fraction of fibres is $5 \%$. On the other hand, discrepancies are greater when the contrast of properties is higher (Fig. 4b, $c_{r}=50$ ). Thus, $\kappa^{*}$ varies from 2.3322 to 2.0730 when $\theta_{m}$ varies from $0^{\circ}$ to $40^{\circ}$ and the volume fraction of fibres is $25 \%$. However, the influence of the tortuosity is still slight when the load rate is low. Indeed, $\kappa^{*}$ varies from 1.1630 to 1.1337 when $\theta_{m}$ varies from $0^{\circ}$ to $40^{\circ}$, the volume fraction of fibres is $5 \%$ and $c_{r}$ is 50 .

\subsection{Width of the interphase area}

Finally, the width of the interphase area is investigated. This one is chosen between 0 for which there is 


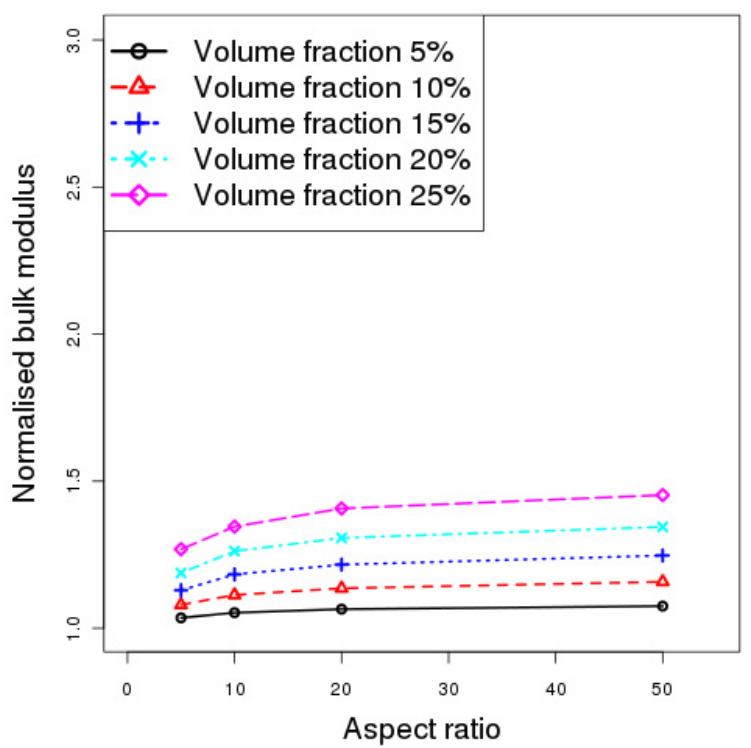

(a)

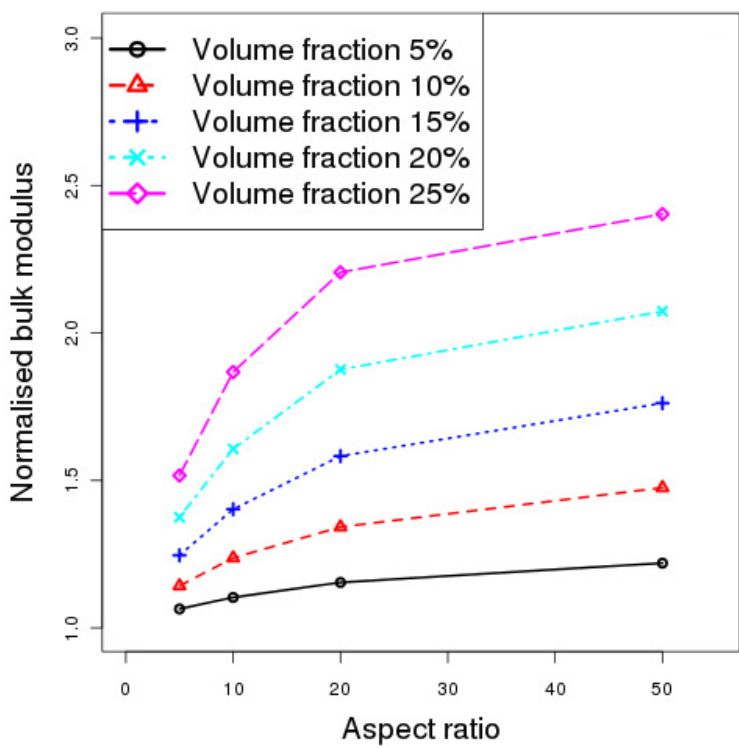

(b)

Fig. 3. Influence of the aspect ratio on the normalised effective bulk modulus (a) $c_{r}=10$, (b) $c_{r}=50$.

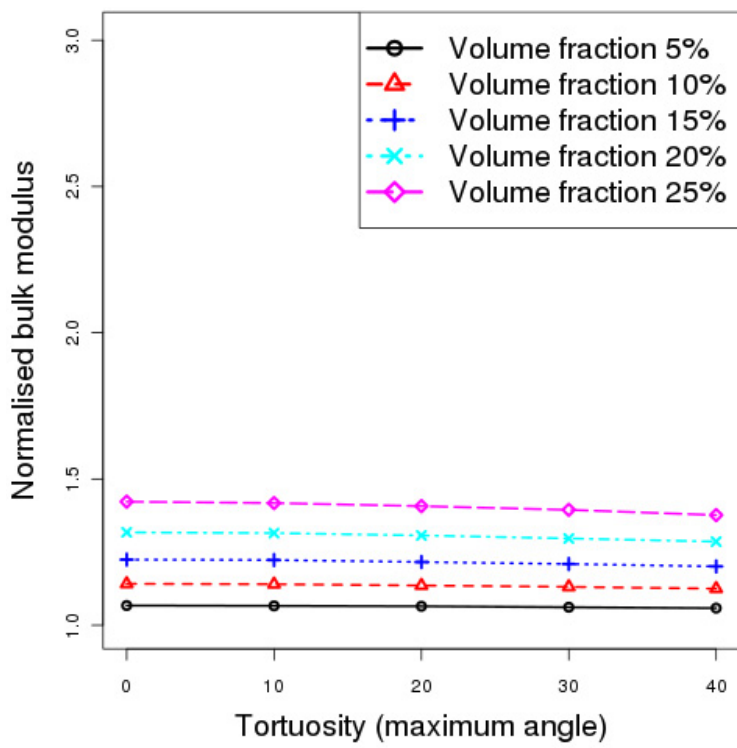

(a)

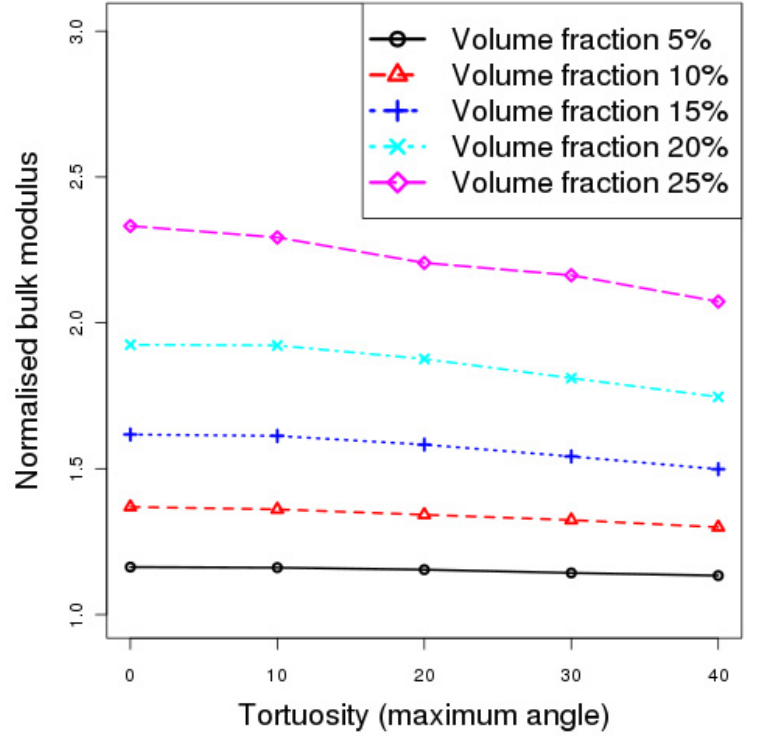

(b)

Fig. 4. Influence of the maximum angle on the normalised effective bulk modulus (a) $c_{r}=10$, (b) $c_{r}=50$.

no interphase area and 0.75 times the diameter of fibres. One must keep in mind that $a_{r}$ is set at $20, \theta_{m}$ is set at 20 and several volume fractions of fibres are considered between 5 and $25 \%$. Figure 5 a shows the influence of the width of the interphase area on $\kappa^{*}$ for a contrast of properties set at 10 . The wider the interphase area is the lower the properties are whatever the load rate of heterogeneities is, which is consistent with the fact that the interphase area is less stiff than the soft phase. Thus, $\kappa^{*}$ varies from 1.5450 to 1.2240 when the volume fraction of reinforcements is $25 \%$ and $w_{\text {int }}$ varies from 0 and $0.75 d$. The same one varies from 1.0907 to 1.0156 when the volume fraction of reinforcements is $5 \%$. In addition, Figure $5 \mathrm{~b}$ depicts the influence of $w_{\text {int }}$ on $\kappa^{*}$ for a contrast of properties set at 50. Results are quite similar to the previous ones. Thus, $\kappa^{*}$ varies from 2.4487 to 1.9358 when the volume fraction of fibres is $25 \%$.

\section{Conclusions}

An investigation of the influence of morphological parameters of a $2 \mathrm{D}$ random short fibre composite on its effective elastic properties has been set up. Conclusions 


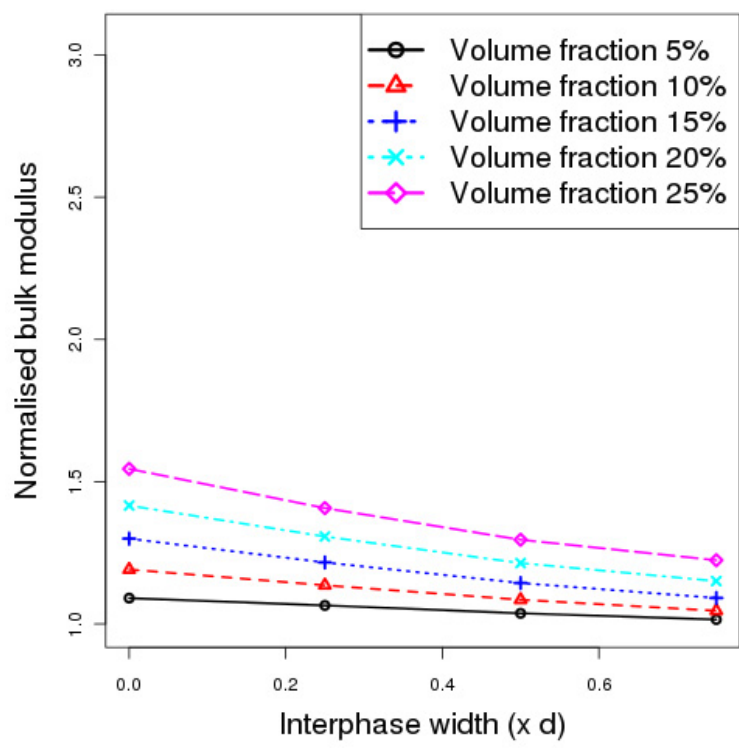

(a)

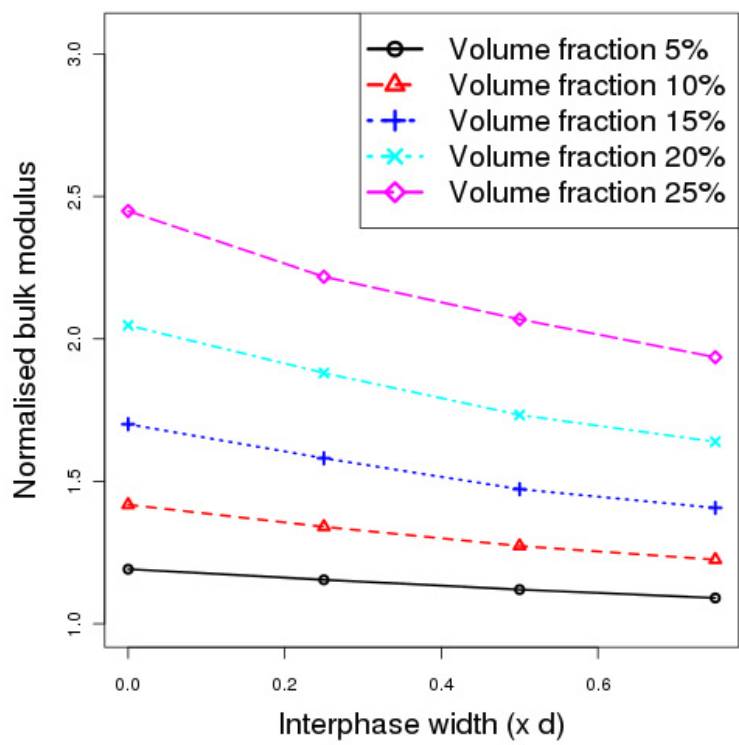

(b)

Fig. 5. Influence of the width of the interphase area on the normalised effective bulk modulus (a) $c_{r}=10$, (b) $c_{r}=50$.

are as follows. First, results confirm the benefit of using long fibres rather than short ones. Second, non-straight fibres slightly affect the mechanical response, especially when both volume fraction of fibres and contrast are high. Finally, the interphase area drastically reduces the reinforcement of the material whatever the contrast of properties is.

Acknowledgements. The authors thank the conseil régional de Basse-Normandie for its financial support to Willy Leclerc's $\mathrm{Ph} . \mathrm{D}$. thesis and the High-Performance Computing Centre of Haute-Normandie (CRIHAN) for the computing means put at their disposal.

\section{References}

[1] T. Mori, K. Tanaka, Average stress in matrix and average elastic energy of materials with misfitting inclusions, Acta Metallurgica 21 (1973) 571-574

[2] R. Hill, A self-consistent mechanics of composite materials, Phys. Solids 13 (1965) 213-222

[3] R.M. Christensen, K.H. Lo, Solutions for effective shear properties in three phase sphere and cylinder models, J. Mech. Phys. Solids 27 (1979) 127-140

[4] S. Torquato, Random heterogeneous media : microstructure and improved bounds on effective properties, Appl. Mech. Rev. 44 (1991) 37-76

[5] J. Segurado, J. Llorca, A numerical approximation to the elastic properties of sphere-reinforced composites, J. Mech. Phys. Solids 50 (2002) 2107-2121
[6] A.A. Gusev, P.J. Hine, I.M. Ward, Fiber packing and elastic properties of a transversely random unidirectional glass/epoxy composite, Compos. Sci. Technol. 60 (2000) $535-41$

[7] H. Moulinec, P. Suquet, A numerical method for computing the overall response of nonlinear composites with complex microstructures, Comput. Methods Appl. Mech. Eng. 157 (1998) 69-94

[8] S. Kari, H. Berger, U. Gabbert, Numerical evaluation of effective material properties of randomly distributed short cylindrical fibre composites, Comput. Mater. Sci. 39 (2007) 198-204

[9] W. Leclerc, P. Karamian, A. Vivet, An efficient stochastic and double-scale model to evaluate the effective elastic properties of $2 \mathrm{D}$ overlapping random fibre composites, Comput. Mater. Sci. 69 (2013) 481-493

[10] F. Dalmas, R. Dendievel, L. Chazeau, J.Y. Cavaillé, C. Gauthier, Carbon nanotube filled polymer composites. Numerical simulation of electrical conductivity in 3D entangled fibrous networks, Acta Materiala 54 (2006) 2923-2931

[11] T. Kanit, S. Forest, I. Galliet, V. Mounoury, D. Jeulin, Determination of the size of the RVE for random composites: statistical and numerical approach, Int. J. Solids Struct. 40(13-14) (2003) 3647-3679

[12] L. Mishnaevsky Jr., K. Derrien, D. Baptiste, Effect of microstructure of particle reinforced composites on the damage evolution: probabilistic and numerical analysis, Compos. Sci. Technol. 64 (2004) 1805-1818

[13] E. Sanchez-Palencia, Non-homogeneous media and vibration theory, Springer, Berlin, 1980 